\title{
Composição das espécies de moluscos bentônicos nos reservatórios do baixo rio Tietê (São Paulo, Brasil) com uma avaliação do impacto causado pelas espécies exóticas invasoras
}

\author{
Roberta S. França; Ana L. Suriani \& Odete Rocha
}

Programa de Pós Graduação em Ecologia e Recursos Naturais, Departamento de Ecologia e Biologia Evolutiva, Universidade Federal de São Carlos. Rodovia Washington Luís km 235, 13565-905 São Carlos, São Paulo, Brasil.

E-mail: robertasebastiany@gmail.com; analuciabio@bol.com.br; doro@power.ufscar.br

\begin{abstract}
Species composition of benthic molluscs in the reservoirs of Low Tietê River (São Paulo, Brazil) with an evaluation of the impact of exotic invader species. The taxonomic composition, density and the spatial distribution of benthic molluscs in low Tietê, São Paulo reservoirs were analyzed in November 2002 and August 2003. The exotic species Melanoides tuberculata was distributed in all portions sampled and was the most abundant species, corresponding to $65.9 \%$ or more of the total abundance, being dominant in all reservoirs, with a maximum density of 23,753 ind. $\mathrm{m}^{-2}$ recorded in the depth of $2 \mathrm{~m}$, at the superior portion of Nova Avanhandava reservoir.The other exotic species, Corbicula fluminea and Helisoma sp., were much less abundant and they were not registered in all portions of the reservoirs. The native species were Aylacostoma tenuilabris, Biomphalaria glabrata, B. intermedia, Physa cubensis and Pomacea canaliculata. These species contributed with less than $20 \%$ of the total abundance of molluscs in the reservoirs, occurring in low densities, much inferior to those of the exotic species, mainly regarding $M$. tuberculata, possibly indicating the impact of the exotic invader species on the native populations.
\end{abstract}

KEY WORDS. Corbicula fluminea; malacofauna; Melanoides tuberculata; Southeast Brazil.

RESUMO. A composição taxonômica, a densidade e a distribuição espacial de moluscos bentônicos nos reservatórios do baixo rio Tietê (São Paulo) foram analisadas. A amostragem dos moluscos foi realizada em novembro de 2002 e agosto de 2003. As características físicas e químicas dos reservatórios também foram avaliadas buscando-se correlacioná-las à composição taxonômica e à distribuição dos táxons através da Análise de Correspondência Canônica (ACC). A espécie exótica Melanoides tuberculata esteve distribuída em todas as porções amostradas e foi a espécie mais abundante, correspondendo a $65,9 \%$ ou mais da abundância total, sendo dominante em todos os reservatórios, com densidade máxima de 23753 ind. $\mathrm{m}^{-2}$ registrada na profundidade de $2 \mathrm{~m}$ da porção superior do reservatório de Nova Avanhandava. As demais espécies exóticas, Corbicula fluminea e Helisoma sp., foram bem menos abundantes e não foram registradas em todas as porções dos reservatórios. As espécies nativas foram Aylacostoma tenuilabris, Biomphalaria glabrata, B. intermedia, Physa cubensis e Pomacea canaliculata. Essas espécies representaram menos de $20 \%$ das espécies presentes nos reservatórios, ocorreram em abundância baixa e em densidades bem inferiores àquelas das espécies exóticas, principalmente em relação a M. tuberculata, podendo indicar o impacto das espécies exóticas invasoras sobre as populações nativas.

PALAVRAS-CHAVE. Corbicula fluminea; malacofauna; Melanoides tuberculata; sudeste do Brasil.

A introdução de espécies exóticas é um dos mais perversos, mais influentes e menos reversíveis dos efeitos humanos em comunidades e no funcionamento dos ecossistemas naturais (SimBERLOFF 1996), sendo que a extinção de espécies nativas é uma das conseqüências mais graves decorrentes da introdução de espécies exóticas invasoras (RICCIARDI \& M ACISAAC 2000). Espécies invasoras são aquelas que além de serem exóticas, caracterizam-se pela rápida maturação sexual, grande capacida- de reprodutora e um considerável poder adaptativo aos ambientes que colonizam, sejam naturais ou artificiais, dulciaqüícolas ou salobros (DARRRIGRAN 1997).

As espécies que colonizam recentemente um ecossistema podem com freqüência causar um impacto negativo sobre o mesmo e alterar seus processos se elas se tornam abundantes e dominam o uso dos recursos (BASKIn 1994). A melhor solução é prevenir a introdução uma vez que a eliminação da população 
invasora é raramente al cançada por três razões: ela é vista como não viável; é cara e pode ter terríveis impactos em espécies nãoalvos (SimberLoff 2001).

As águas doces são ambientes particularmente sujeitos às invasões por espécies exóticas, pois a dispersão destas é grandemente facilitada pelo próprio fluxo de água. WELCOMmE (1988), por exemplo, verificou 1354 introduções de espécies exóticas invasoras em cerca de 140 países.

Entre os grupos de invertebrados de água doce com elevado potencial de invasão, os moluscos se destacam pelo sucesso com que têm invadido as águas doces em todo o mundo, como é o caso das espécies de bivalvos exóticos e invasores Dreissena polymorpha (Pallas, 1771) (Dreissenidae) (“mexiIhão zebra"), Limnoperna fortunei (Dunker, 1857) (Mytilidae) ("mexilhão dourado") Corbicula fluminea (Muller, 1774) (Corbiculidae) ("Asia clam") (VAz et al. 1986, Johnson \& CARLton 1996, StRAYer 1999) e do gatrópode Melanoides tuberculata (Müller 1774) (Thiaridae). O número de introduções têm aumentado nos últimos anos em decorrência das novas tecnologias e a globalização que aceleraram a movimentação de organismos ao redor do mundo (RICCIARDI \& MACISAAC 2000).

No Brasil, M. tuberculata e $C$. fluminea merecem destaque pela recente invasão e estabelecimento em diversas bacias hidrográficas. M. tuberculata é nativo do leste da África, sudeste da Ásia, China e Ilhas do Indo-Pacífico, com uma ampla distribuição nessas áreas (PoInTIER et al. 1993). No Brasil esta espécie foi registrada pela primeira vez na cidade de Santos (São Paulo), em 1967 (VAZ et al. 1986), e sua introdução foi provavelmente associada ao comércio de plantas e peixes ornamentais (Fernandez et al. 2003). É capaz de colonizar uma ampla variedade de habitats, em corpos d'água de diversos graus de trofia, desde águas levemente salinas, até ambientes oligohalinos, até $1.500 \mathrm{~m}$ de altitude, sendo ainda resistentes a baixas concentrações de oxigênio dissolvido (DUDGEON, 1986).

Corbicula fluminea é originário da China, tendo sido registrado pela primeira vez na América do Sul, na década de 70, no rio da Prata (ITUARTE 1981) e no final da década de 1960 no Brasil, (Veitenheimer-Mendes 1981). É considerado um grave problema nos Estados Unidos em relação a ambos impactos, econômicos e ecológicos (CATALdo \& Boltovskoy 1999).

O presente estudo teve por objetivo analisar a composição taxonômica, a densidade e a distribuição espacial e temporal dos moluscos bentônicos em três reservatórios em cascata, na porção do baixo rio Tietê (Promissão, Nova Avanhandava e Três Irmãos), visando obter informações sobre a presença e o impacto das espécies exóticas invasoras nestes sistemas.

\section{MATERIAL E MÉTODOS}

Para a caracterização da malacofauna dos reservatórios do baixo rio Tietê, amostragens da comunidade bentônica foram realizadas nos reservatórios de Promissão, Nova Avanhandava e Três Irmãos (Fig. 1). Estes reservatórios Iocalizam-se no estado de São Paulo sofrendo atualmente forte pressão antrópica, em decorrência da elevada densidade populacional e da alta atividade industrial (Tundısı et al. 1991).

A área de drenagem do baixo rio Tietê é de aproximadamente $13.655 \mathrm{~km}^{2}$ e abrange cerca de 32 municípios. A água é utilizada para abastecimento, navegação e disposição de efluentes domésticos e industriais. Além das atividades econômicas tradicionais (cana de açúcar, indústria têxtil), ocorrem extensas áreas de pastagens cultivadas (CETESB 2001). As características morfométricas e operacionais dos reservatórios estão representadas na tabela I, de acordo com CESP (1998).

As coletas foram realizadas em novembro de 2002 (período chuvoso) e agosto de 2003 (período seco) estabelecendo-se três transectos em cada reservatório: porção superior, mediana e inferior (barragem), com três a seis pontos amostrais em cada transecto.

As variáveis físicas e químicas da água e a determinação da concentração de nutrientes seguiram a metodologia de GolteRMAN et al. (1978) e M ACKERETH et al. (1978). A composição granulométrica do sedimento foi determinada segundo a metodologia descrita por Suguio (1973).

As amostras de sedimento foram coletadas com draga do tipo van Veen e os moluscos foram identificados até o nível de espécie de acordo com Thompson (2004) e com o auxílio de especialistas Dr. Wagner E. P. Avelar da Faculdade de Filosofia, Ciências e Letras de Ribeirão Preto (USP) (para os bivalvos) e Dr. Luiz Ricardo Lopez de Simone, do Museu de Zoologia da Universidade de São Paulo (para os gastrópodos). A densidade dos organismos foi calculada de acordo com WeLCH (1948).

Realizou-se uma análise de regressão múltipla passo a passo - Stepwise multiple regression (programa NCSSPC) para verificar as possíveis correlações entre as variações na abundância das espécies e as variáveis abióticas. Por meio da análise de variância multivariada - MANOVA (programa SYSTAT) realizada sobre as matrizes de dados abióticos (períodos chuvoso e seco) testou-se o efeito da sazonalidade com nível de corte de probabilidade de $5 \%$. O passo seguinte foi preparar duas novas matrizes de dados bióticos e abióticos para aplicação da análise de correspondência canônica (ACC) (Programa MVSP 3.1), considerando-se os períodos de coleta.

\section{RESULTADOS}

\section{Variáveis abióticas}

A temperatura média na superfície da água das três represas evidenciou a variação sazonal, com temperaturas mais elevadas no período chuvoso. $\mathrm{O}$ pH foi aproximadamente neutro, com faixa de variação de 6,6 a 8,6 no período chuvoso, valores em média ligeiramente menores do que aqueles registrados no período seco. A coluna de água dos reservatórios esteve bem oxigenada, em ambos os períodos, com valores médios compreendidos entre 6,7 e 10,7 mg.l-1. Nos três reservatórios amostrados a condutividade foi mais elevada no período chuvoso (Tab. II).

Revista Brasileira de Zoologia 24 (1): 41-51, março 2007 


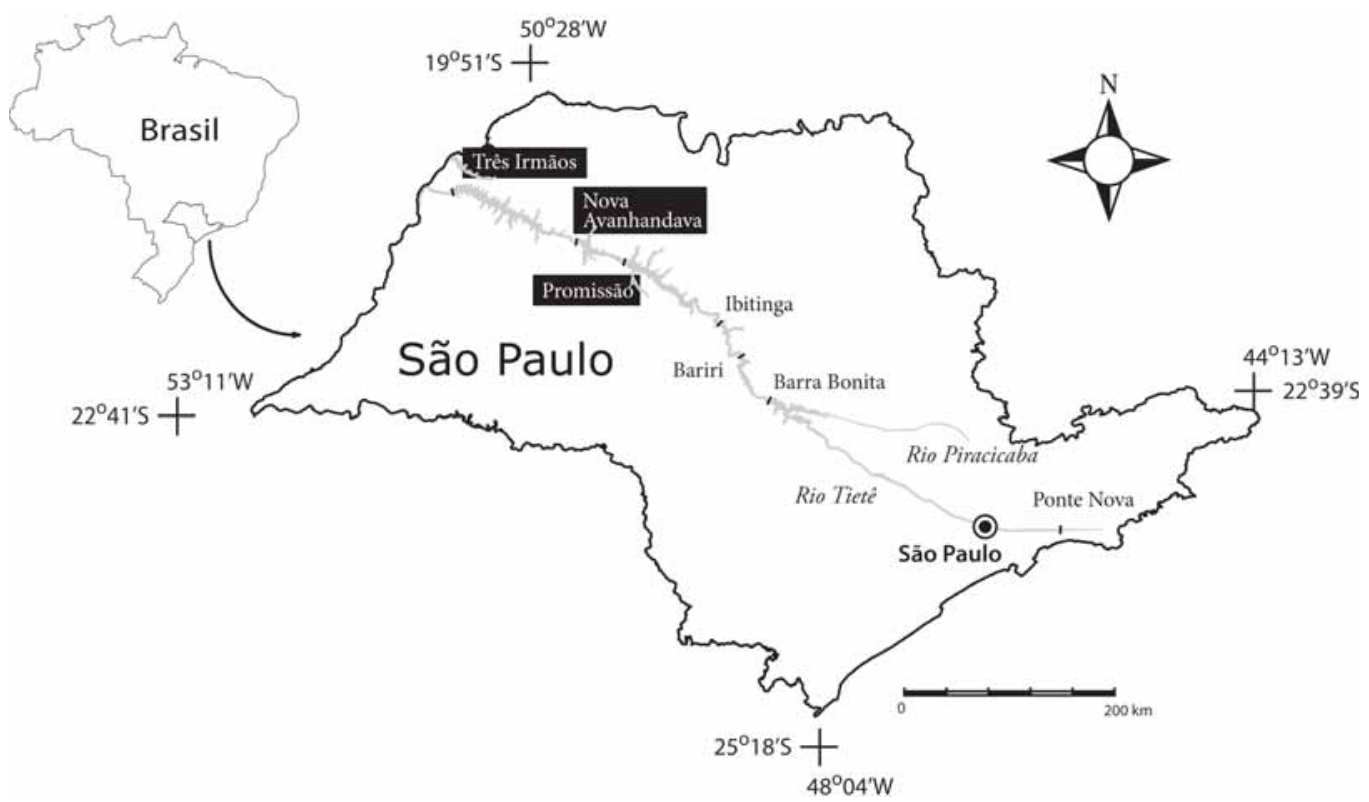

Figura 1. Localização do Sistema Tietê no estado de São Paulo e dos principais reservatórios dos rios Tietê e Paraná, evidenciando a localização dos reservatórios de Promissão, Nova Avanhandava e Três Irmãos. Modificado de RocHA et al. (2006).

Tabela I. Características morfométricas e operacionais dos reservatórios do baixo rio Tietê.

\begin{tabular}{lccccc}
\hline \multicolumn{1}{c}{ Reservatórios } & Tempo de residência (dias) & Área $\left(\mathrm{km}^{2}\right)$ & Volume $\left(\mathrm{m}^{3} \times \mathrm{106}\right)$ & Profundidade média $(\mathrm{m})$ & Ano de enchimento \\
\hline Promissão & 134,1 & 530 & 7408 & 14,0 & 1975 \\
Nova Avanhandava & 45,7 & 210 & 2720 & 13,0 & 1985 \\
Três Irmãos & 217,9 & 817 & 14000 & 17,2 & 1991 \\
\hline
\end{tabular}

Tabela II. Valores médios das variáveis físicas e químicas da água nas porções superior, mediana e inferior (barragem) dos reservatórios de Promissão, Nova Avanhandava e Três Irmãos, em novembro de 2002 e agosto de 2003.

\begin{tabular}{|c|c|c|c|c|c|c|c|}
\hline \multirow{2}{*}{ Localidade } & \multirow{2}{*}{ Variável } & \multicolumn{2}{|c|}{ Superior } & \multicolumn{2}{|c|}{ Mediana } & \multicolumn{2}{|c|}{ Inferior } \\
\hline & & Nov/02 & Ago/03 & Nov/02 & Ago/03 & Nov/02 & Ago/03 \\
\hline \multirow[t]{4}{*}{ Promissão } & $\mathrm{pH}$ & 6,9 & 7,9 & 6,6 & 7,1 & 8,6 & 7,6 \\
\hline & Cond. $\left(\mu \mathrm{S} . \mathrm{cm}^{-1}\right)$ & 273,0 & 151,0 & 218,8 & 128,5 & 135,3 & 135,7 \\
\hline & $\mathrm{OD}\left(\mathrm{mg} . \mathrm{l}^{-1}\right)$ & 10,3 & 8,9 & 9,7 & 7,3 & 8,5 & 8,1 \\
\hline & $\mathrm{T}\left({ }^{\circ} \mathrm{C}\right)$ & 28,2 & 21,8 & 27,1 & 20,3 & 29,2 & 20,5 \\
\hline \multirow[t]{4}{*}{ Nova Avanhandava } & $\mathrm{pH}$ & 8,4 & 7,8 & 8,6 & 7,5 & 7,4 & 8,1 \\
\hline & Cond. $\left(\mu \mathrm{S} . \mathrm{cm}^{-1}\right)$ & 131,7 & 128,7 & 133,0 & 139,6 & 163,5 & 149,8 \\
\hline & $\mathrm{OD}\left(\mathrm{mg} \cdot \mathrm{I}^{-1}\right)$ & 7,8 & 7,9 & 7,6 & 7,9 & 10,7 & 9,0 \\
\hline & $\mathrm{T}\left({ }^{\circ} \mathrm{C}\right)$ & 26,9 & 20,4 & 26,8 & 20,2 & 27,2 & 21,2 \\
\hline \multirow[t]{4}{*}{ Três Irmãos } & $\mathrm{pH}$ & 7,4 & 7,5 & 7,5 & 7,8 & 7,1 & 7,8 \\
\hline & Cond. $\left(\mu \mathrm{S} . \mathrm{cm}^{-1}\right)$ & 163,3 & 150,8 & 139,0 & 146,3 & 133,3 & 124,8 \\
\hline & $\mathrm{OD}\left(\mathrm{mg} . \mathrm{I}^{-1}\right)$ & 8,6 & 8,6 & 6,7 & 9,1 & 7,3 & 8,2 \\
\hline & $\mathrm{T}\left({ }^{\circ} \mathrm{C}\right)$ & 27,5 & 20,1 & 26,7 & 21,7 & 26,3 & 20,9 \\
\hline
\end{tabular}

Uma ampla faixa de variação foi observada em relação ás concentrações médias dos compostos nitrogenados e fosfatados, e com exceção do íon amônio, ocorreram concentrações mais elevadas no período chuvoso (Tab. III). A análise da composição 
Tabela III. Valores médios das concentrações dos nutrientes dissolvidose totais da água nas porções superior, mediana e inferior (barragem) dos reservatórios de Promissão, Nova Avanhandava e Três Irmãos, em novembro de 2002 e agosto de 2003. (NT) Nitrogênio total, (FID) fosfato inorgânico dissolvido, (FTD) fósforo total dissolvido, (FT) fósforo total.

\begin{tabular}{|c|c|c|c|c|c|c|c|}
\hline \multirow{2}{*}{ Localidade } & \multirow{2}{*}{ Variável } & \multicolumn{2}{|c|}{ Superior } & \multicolumn{2}{|c|}{ Mediana } & \multicolumn{2}{|c|}{ Inferior } \\
\hline & & Nov/02 & Ago/03 & Nov/02 & $\mathrm{Ago} / 03$ & Nov/02 & $\mathrm{Ago} / 03$ \\
\hline \multirow[t]{7}{*}{ Promissão } & Nitrito $\left(\mu \mathrm{g} .\left.\right|^{-1}\right)$ & 15,31 & 5,28 & 9,25 & 5,92 & 2,33 & 1,17 \\
\hline & Nitrato $\left(\mu \mathrm{g} . \mathrm{I}^{-1}\right)$ & 1450,07 & 521,43 & 715,90 & 315,38 & 280,70 & 276,66 \\
\hline & Amônio $\left(\mu \mathrm{g} . \mathrm{I}^{-1}\right)$ & 17,31 & 18,98 & 7,88 & 34,92 & 10,34 & 19,11 \\
\hline & NT (mg. $\left.\left.\right|^{-1}\right)$ & 1,08 & 0,89 & 0,98 & 0,39 & 1,04 & 0,30 \\
\hline & FID $\left(\mu \mathrm{g} . \mathrm{I}^{-1}\right)$ & 7,40 & 2,50 & 6,84 & 2,45 & 5,93 & 2,94 \\
\hline & $\mathrm{FTD}\left(\mu \mathrm{g} . \mathrm{I}^{-1}\right)$ & 18,68 & 11,67 & 16,51 & 9,22 & 12,78 & 8,00 \\
\hline & $\mathrm{FT}\left(\mu \mathrm{g} \cdot .^{-1}\right)$ & 95,21 & 33,83 & 87,15 & 37,65 & 74,72 & 35,91 \\
\hline \multirow[t]{7}{*}{ Nova Avanhandava } & Nitrito $\left(\mu \mathrm{g} . \mathrm{I}^{-1}\right)$ & 3,16 & 0,98 & 5,11 & 1,62 & 2,12 & 1,92 \\
\hline & Nitrato $\left(\mu \mathrm{g} . \mathrm{I}^{-1}\right)$ & 264,38 & 171,66 & 233,65 & 254,46 & 170,38 & 220,38 \\
\hline & Amônio $\left(\mu \mathrm{g} . \mathrm{I}^{-1}\right)$ & 13,80 & 18,59 & 11,01 & 31,11 & 17,90 & 22,22 \\
\hline & NT (mg. $\left.\left.\right|^{-1}\right)$ & 1,08 & 0,50 & 0,94 & 0,65 & 1,40 & 0,69 \\
\hline & FID $\left(\mu g . I^{-1}\right)$ & 2,60 & 2,70 & 5,34 & 6,10 & 3,65 & 3,28 \\
\hline & $\operatorname{FTD}\left(\mu \mathrm{g} . \mathrm{I}^{-1}\right)$ & 11,38 & 7,59 & 13,25 & 18,23 & 11,20 & 8,20 \\
\hline & $\mathrm{FT}\left(\mu \mathrm{g} . \mathrm{I}^{-1}\right)$ & 101,76 & 17,82 & 92,61 & 23,87 & 124,62 & 33,85 \\
\hline \multirow[t]{7}{*}{ Três Irmãos } & Nitrito $\left(\mu \mathrm{g} . \mathrm{I}^{-1}\right)$ & 3,15 & 1,54 & 3,38 & 0,52 & 2,60 & 1,38 \\
\hline & Nitrato $\left(\mu \mathrm{g} . \mathrm{I}^{-1}\right)$ & 128,73 & 168,40 & 193,29 & 143,88 & 171,67 & 129,56 \\
\hline & Amônio $\left(\mu \mathrm{g} . \mathrm{I}^{-1}\right)$ & 18,00 & 23,84 & 27,42 & 14,19 & 28,87 & 16,13 \\
\hline & NT (mg. $\left.\left.\right|^{-1}\right)$ & 2,66 & 0,20 & 0,93 & 0,20 & 0,54 & 0,35 \\
\hline & FID $\left(\mu \mathrm{g} . \mathrm{I}^{-1}\right)$ & 4,59 & 2,84 & 5,44 & 2,30 & 5,26 & 3,18 \\
\hline & $\operatorname{FTD}\left(\mu \mathrm{g} . \mathrm{I}^{-1}\right)$ & 12,61 & 7,21 & 12,15 & 6,22 & 13,72 & 7,54 \\
\hline & $\mathrm{FT}\left(\mu \mathrm{g} . \mathrm{I}^{-1}\right)$ & 123,91 & 23,11 & 136,33 & 21,79 & 108,09 & 23,36 \\
\hline
\end{tabular}

dos sedimentos dos reservatórios revel ou que a areia foi a fração granulométrica predominante, seguida pela argila e silte, em ambos os períodos amostrados. O conteúdo médio de matéria orgânica representou no máximo 10,59\% da composição do sedimento, considerando-se os períodos amostrados (Tab. IV).

Variáveis bióticas

A tabela $V$ contém a abundância relativa (\%) de moluscos na porção superior, mediana e inferior (barragem) dos reservatórios de Promissão, Nova Avanhandava e Três Irmãos, em novembro de 2002 e agosto de 2003. A malacofauna registrada nos reservatórios do baixo rio Tietê foi composta por cinco espécies nativas, Aylacostoma tenuilabris (Bernardi, 1856) (Thiaridae), Biomphalaria glabrata (Say, 1818), B. intermedia (Paraense $\&$ Deslandes, 1962) (Planorbidae), Physa cubensis (Pfeiffer, 1839) (Physidae) e Pomacea canaliculata (Lamarck, 1822) (Ampullariidae), e três espécies exóticas que representaram mais de $80 \%$ das espécies de moluscos presentes nos reservatórios: M elanoides tuberculata, Corbicula fluminea e Helisoma sp. (Swainson, 1840) (Planorbidae).

Entre as espécies nativas, as espécies Biomphalaria glabrata e $B$. intermedia foram às únicas que ocorreram em todas as porções dos reservatórios (Figs 2 a 7). A espécie $A$. tenuilabris não foi registrada nas porções inferior e mediana do reservatório de Três Irmãos, em ambos os períodos de amostragem (Figs 6 e 7) e P. canaliculata foi registrada somente no período seco na porção superior da represa de Promissão (Fig. 3) e na porção mediana do reservatório de Nova Avanhandava (Fig. 5), porém em densidades muito baixa, não ultrapassando 27 ind. $\mathrm{m}^{-2}$. Physa cubensis somente não foi observada na porção inferior do reservatório de Promissão, em ambos os períodos de amostragem e na porção mediana deste mesmo reservatório no período seco (Figs 2 e 3). As maiores densidades registradas para essa espécie foram de 4.350 ind. $\mathrm{m}^{-2}$ no período chuvoso (Fig. 4) e 1631 ind. $\mathrm{m}^{-2}$ no período seco (Fig. 7) nas porções superiores dos reservatórios de Nova Avanhandava (profundidade de 2 m) e Três Irmãos (profundidade de $1 \mathrm{~m}$ ), respectivamente. A abundância relativa máxima $(5,4 \%)$ registrada para essa espécie foi no período seco, na porção superior do reservatório de Nova Avanhandava.

A densidade máxima em que $A$. tenuilabris ocorreu nos reservatórios foi de 663 ind. $\mathrm{m}^{-2}$, no período seco, na profundidade de $5 \mathrm{~m}$ da porção superior do reservatório de Nova Avanhandava (Fig. 5). A abundância relativa máxima registrada para essa espécie $(9,2 \%)$ foi no período seco, na porção mediana do reservatório de Promissão. Biomphalaria spp. foi re- 

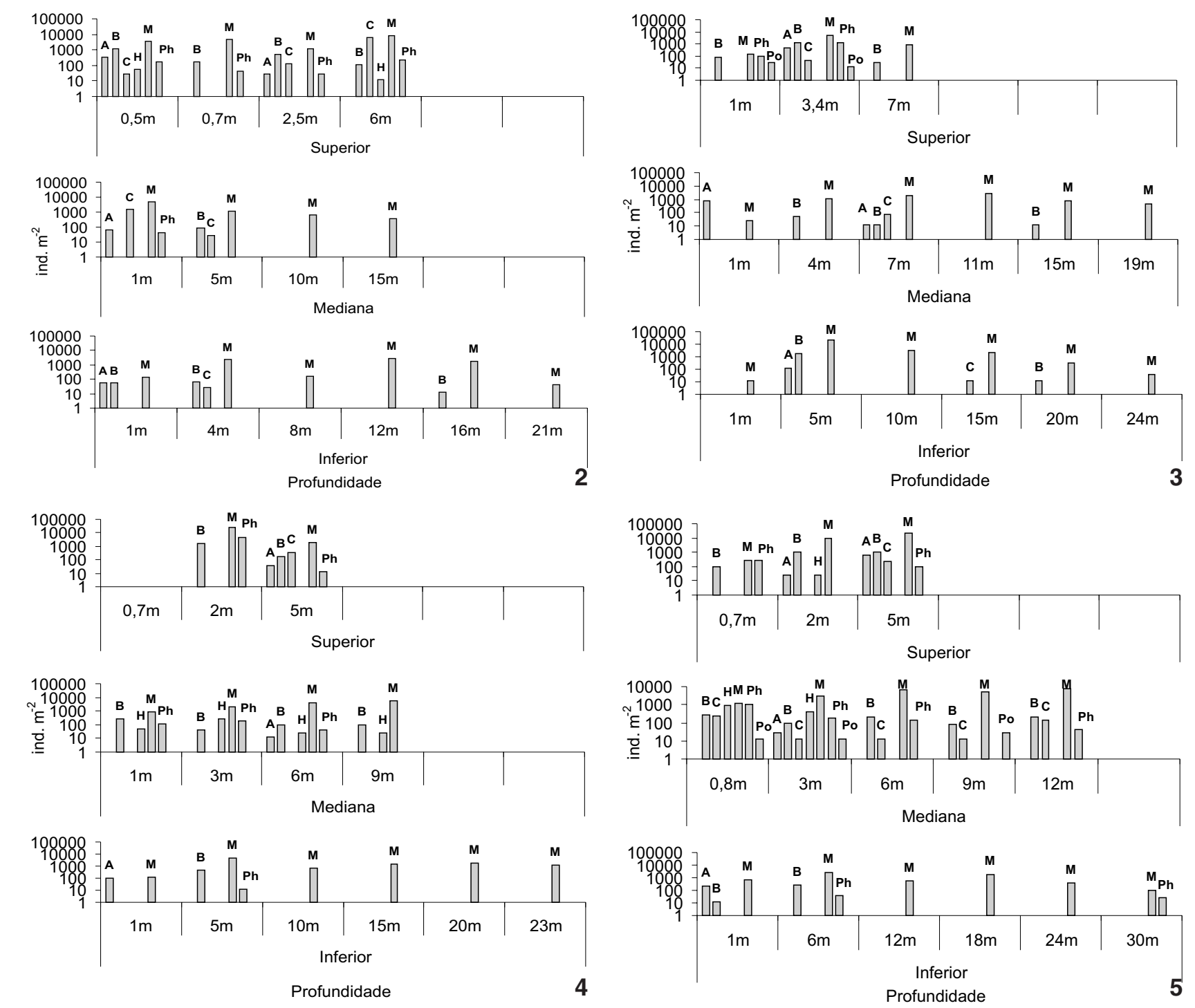

Figuras 2-5. Variação da densidade (ind. $\mathrm{m}^{-2}$ ), em escala logarítmica, dos diferentes táxons de moluscos ( $\mathrm{A}$, Aylacostoma tenuilabris, $\mathrm{B}$, Biomphalaria glabrata, C, Corbicula fluminea H, (Helisoma sp.), Ph, Physa cubensis e Po, Pomacea canaliculata) em relação à profundidade (m), na porção superior, mediana e inferior (barragem) do reservatório de: (2) Promissão, em novembro de 2002; (3) Promissão, em agosto de 2003; (4) Nova Avanhandava, em novembro de 2002; (5)Nova Avanhandava, em agosto de 2003.

gistrada com uma densidade máxima de 1.565 ind. $\mathrm{m}^{-2}$, no período chuvoso, na profundidade de $2 \mathrm{~m}$ da porção superior do reservatório de Nova Avanhandava (Fig. 4). A mais elevada abundância relativa $(14,4 \%)$ foi registrada no período seco, na porção superior do reservatório de Promissão.

Em relação às espécies exóticas presentes nos reservatórios, o gênero Helisoma foi o que se mostrou com menor distribuição, ocorrendo com densidade máxima de 1233 ind.m-2 (profundidade de $1 \mathrm{~m}$ ), no período seco, na porção superior do reservatório de Três Irmãos (Fig. 7). Corbicula fluminea foi regis- trado no período chuvoso com densidade máxima de 6154 ind. $\mathrm{m}^{-2}$ na porção superior (profundidade de $6 \mathrm{~m}$ ) do reservatório de Promissão (Fig. 2). Nas porções inferiores dos reservatórios de Nova Avanhandava e de Três I rmãos não houve registro da ocorrência dessa espécie, em ambos os períodos de coleta (Figs 4 a 7). No reservatório de Promissão esta espécie foi mais abundante no período chuvoso, na porção superior, representando $23,7 \%$ dos indivíduos.

A espécie M. tuberculata esteve presente em quase todas as profundidades amostradas e ocorreu em densidades bem 

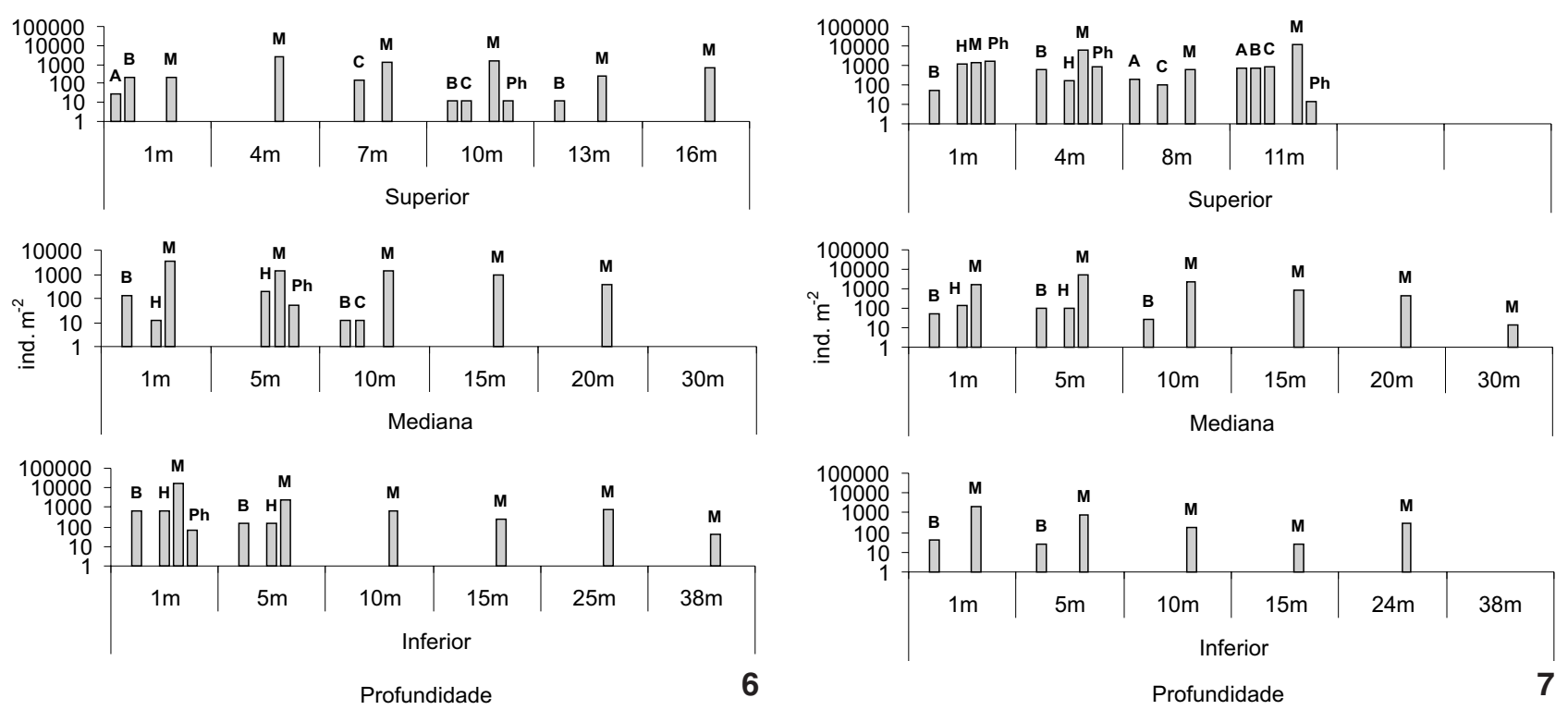

Figuras 6-7. Variação da densidade (ind. $\mathrm{m}^{-2}$ ), em escala logarítmica, dos diferentes táxons de moluscos (A, Aylacostoma tenuilabris, B, Biomphalaria glabrata, C, Corbicula fluminea H, (Helisoma sp.), Ph, Physa cubensis e Po, Pomacea canaliculata) em relação à profundidade (m), na porção superior, mediana e inferior (barragem) do reservatório de Três Irmãos, em novembro de 2002 (6) e em agosto de 2003 (7).

Tabela IV. Valores médios (em porcentagem) das frações granulométricas do sedimento dos reservatórios de Promissão, Nova Avanhandava e Três Irmãos, nas porções superior, mediana e inferior (barragem), em novembro de 2002 e agosto de 2003.

\begin{tabular}{|c|c|c|c|c|c|c|c|}
\hline \multirow{2}{*}{ Localidade } & \multirow{2}{*}{ Variável } & \multicolumn{2}{|c|}{ Superior } & \multicolumn{2}{|c|}{ Mediana } & \multicolumn{2}{|c|}{ Inferior } \\
\hline & & Nov/02 & $\mathrm{Ago} / 03$ & Nov/02 & $\mathrm{Ago} / 03$ & Nov/02 & $\mathrm{Ago} / 03$ \\
\hline \multirow[t]{4}{*}{ Promissão } & Areia & 79,8 & 73,4 & 65,5 & 64,9 & 68,0 & 62,5 \\
\hline & Silte & 6,4 & 10,4 & 11,6 & 14,2 & 7,9 & 12,9 \\
\hline & Argila & 13,8 & 16,2 & 22,9 & 20,9 & 24,1 & 24,6 \\
\hline & Matéria orgânica & 2,2 & 7,0 & 2,7 & 3,1 & 4,0 & 4,0 \\
\hline \multirow[t]{4}{*}{ Nova Avanhandava } & Areia & 57,3 & 75,3 & 43,6 & 49,8 & 59,0 & 74,3 \\
\hline & Silte & 13,0 & 8,7 & 14,9 & 18,0 & 17,5 & 5,8 \\
\hline & Argila & 29,7 & 16,0 & 41,6 & 32,2 & 23,6 & 19,9 \\
\hline & Matéria orgânica & 1,4 & 2,9 & 7,3 & 6,7 & 7,3 & 3,3 \\
\hline \multirow[t]{4}{*}{ Três Irmãos } & Areia & 31,0 & 90,3 & 90,9 & 76,2 & 48,5 & 52,6 \\
\hline & Silte & 16,7 & 2,6 & 1,5 & 4,4 & 18,5 & 14,8 \\
\hline & Argila & 52,3 & 7,1 & 7,5 & 19,1 & 33,0 & 32,4 \\
\hline & Matéria orgânica & 4,7 & 1,5 & 1,5 & 4,2 & 9,8 & 10,6 \\
\hline
\end{tabular}

elevadas, em ambos os períodos de coleta, com abundância relativa superior a $65,9 \%$. No período chuvoso, foram registrados valores extremos de 23.753 ind. $\mathrm{m}^{-2}$ na profundidade de $2 \mathrm{~m}$ da porção superior do reservatório de Nova Avanhandava (Fig. 4) e 17.042 ind. $\mathrm{m}^{-2}$ na profundidade de $1 \mathrm{~m}$ na porção inferior (barragem) do reservatório de Três Irmãos (Fig. 6). No período seco, os maiores valores de densidade para essa espécie foram obtidos na profundidade de $5 \mathrm{~m}$ da porção superior do reservatório de Nova Avanhandava, onde se verificou uma densidade de 20570 ind. $\mathrm{m}^{-2}$ (Fig. 5) e na profundidade de $5 \mathrm{~m}$ da porção inferior do reservatório de Promissão com 23448 ind. $\mathrm{m}^{-2}$ (Fig. 3).

Os resultados obtidos por meio da análise de regressão múltipla passo a passo não indicaram correlação significativa entre a abundância dos moluscos e as variáveis abióticas medidas nesse trabalho. A análise multivariada (MANOVA) revelou que houve variação significativa dos fatores físicos e químicos da água entre os períodos seco e chuvoso, evidenciando a existência de sazonalidade ( $p<0,05)$, expressas principal mente pelas variáveis temperatura, concentração de oxigênio dissolvido e de nutrientes. 
Tabela V. Abundância relativa (\%) de moluscos na porção superior, mediana e inferior (barragem) dos reservatórios de Promissão, Nova Avanhandava e Três Irmãos, em novembro de 2002 e agosto de 2003.

\begin{tabular}{|c|c|c|c|c|c|c|c|}
\hline \multirow{2}{*}{ Localidade } & \multirow{2}{*}{ Táxons } & \multicolumn{2}{|c|}{ Superior } & \multicolumn{2}{|c|}{ Mediana } & \multicolumn{2}{|c|}{ Inferior } \\
\hline & & Nov/02 & $\mathrm{Ago} / 03$ & Nov/02 & Ago/03 & Nov/02 & Ago/03 \\
\hline \multirow[t]{7}{*}{ Promissão } & Aylacostoma tenuilabris & 1,3 & 5,2 & 0,7 & 9,2 & 0,7 & 0,4 \\
\hline & Biomphalaria spp. & 7,2 & 14,4 & 1,0 & 1,0 & 1,7 & 6,3 \\
\hline & Corbicula fluminea & 23,7 & 0,4 & 18,0 & 1,0 & 0,3 & - \\
\hline & Helisoma sp. & 0,2 & - & - & - & - & - \\
\hline & M elanoides tuberculata & 65,9 & 66,1 & 79,8 & 88,8 & 97,3 & 93,3 \\
\hline & Physa cubensis & 1,6 & 13,5 & 0,4 & - & - & - \\
\hline & Pomacea canaliculata & - & 0,4 & - & - & - & - \\
\hline \multirow[t]{7}{*}{ Nova Avanhandava } & Aylacostoma tenuilabris & 0,1 & 2,1 & 0,1 & 0,1 & 0,9 & 3,0 \\
\hline & Biomphalaria spp. & 5,4 & 6,4 & 3,4 & 3,1 & 5,1 & 4,0 \\
\hline & Corbicula fluminea & 1,2 & 0,7 & - & 1,4 & - & - \\
\hline & Helisoma sp. & - & 0,1 & 2,6 & 4,6 & - & - \\
\hline & Melanoides tuberculata & 79,8 & 89,7 & 91,5 & 85,7 & 93,9 & 92,1 \\
\hline & Physa cubensis & 13,5 & 1,0 & 2,4 & 5,0 & 0,1 & 1,0 \\
\hline & Pomacea canaliculata & - & - & - & 0,2 & - & - \\
\hline \multirow[t]{7}{*}{ Três Irmãos } & Aylacostoma tenuilabris & 0,4 & 3,2 & - & - & - & - \\
\hline & Biomphalaria spp. & 3,5 & 5,4 & 1,8 & 1,6 & - & 1,8 \\
\hline & Corbicula fluminea & 2,4 & 3,7 & 0,2 & - & - & - \\
\hline & Helisoma sp. & - & 5,4 & 2,5 & 2,2 & 3,5 & 1,1 \\
\hline & M elanoides tuberculata & 93,5 & 72,8 & 94,8 & 96,2 & 96,2 & 97,1 \\
\hline & Physa cubensis & 0,2 & 9,5 & 0,7 & - & 0,3 & - \\
\hline & Pomacea canaliculata & - & - & - & - & - & - \\
\hline
\end{tabular}

A análise de correspondência canônica (ACC) para os dados obtidos no período chuvoso explicou 94,33\% da variância total dos dados (Fig. 8). O eixo 1 explicou 67,3\% da variância total dos dados e esteve positivamente relacionado com elevados valores de temperatura (T), oxigênio dissolivdo (Oxig) e fósforo total dissolvido (PTD) que caracterizaram as porções superior e mediana do reservatório de Promissão, onde as espé cies $A$. tenuilabris e $C$. fluminea ocorreram com maior abundância. Quando comparadas às demais espécies, C. fluminea, esteve associada a ambientes mais oxigenados.

O eixo 2 explicou $27 \%$ da variância total dos dados e esteve positivamente relacionado com elevadas concentrações de nitrogênio total (NT), onde se observou uma associação das espécies do gênero Biomphalaria e elevados valores de pH caracterizando a porção superior do reservatório de Nova Avanhandava onde se observou elevada abundância da espécie P. cubensis. Em oposição, projetam-se negativamente ao eixo 2 concentrações elevadas de amônio (NH4) e concentrações intermediárias de matéria orgânica (MO) que estiveram associadas ao reservatório de Três Irmãos, à porção mediana e inferior do reservatório de
Nova Avanhandava e à porção inferior do reservatório de Promissão, onde se observou elevada abundância de Helisoma sp.

A análise de correspondência canônica (CCA) para o período seco explicou $83,29 \%$ da variância total dos dados (Fig. 9). O eixo 1 explicou $59,25 \%$ da variância total dos dados e esteve positivamente relacionado com elevadas concentrações de nitrito (NO2) na porção superior do reservatório de Promissão, onde se observou reduzida abundância das espécies P. cubensis eP. canaliculata. Em oposição, projeta-se negativamente ao eixo 1 concentrações intermediárias de condutividade na porção superior do reservatório de Três Irmãos e na porção mediana de Nova Avanhandava, onde se verificou uma associação das espécies $C$. fluminea e Helisoma sp.

O eixo 2 explicou 24,04\% da variância total dos dados, sendo relacionado positivamente com elevadas concentrações de nitrato (NO3) e nitrogênio total (NT) que se associaram com reduzida abundância das espécies $B$. glabrata, B. intermedia e A. tenuilabris. As demais espécies não se associaram com nenhuma das variáveis neste eixo. Em ambos os períodos amostrados a espécie exótica M. tuberculata foi à única que não esteve asso- 

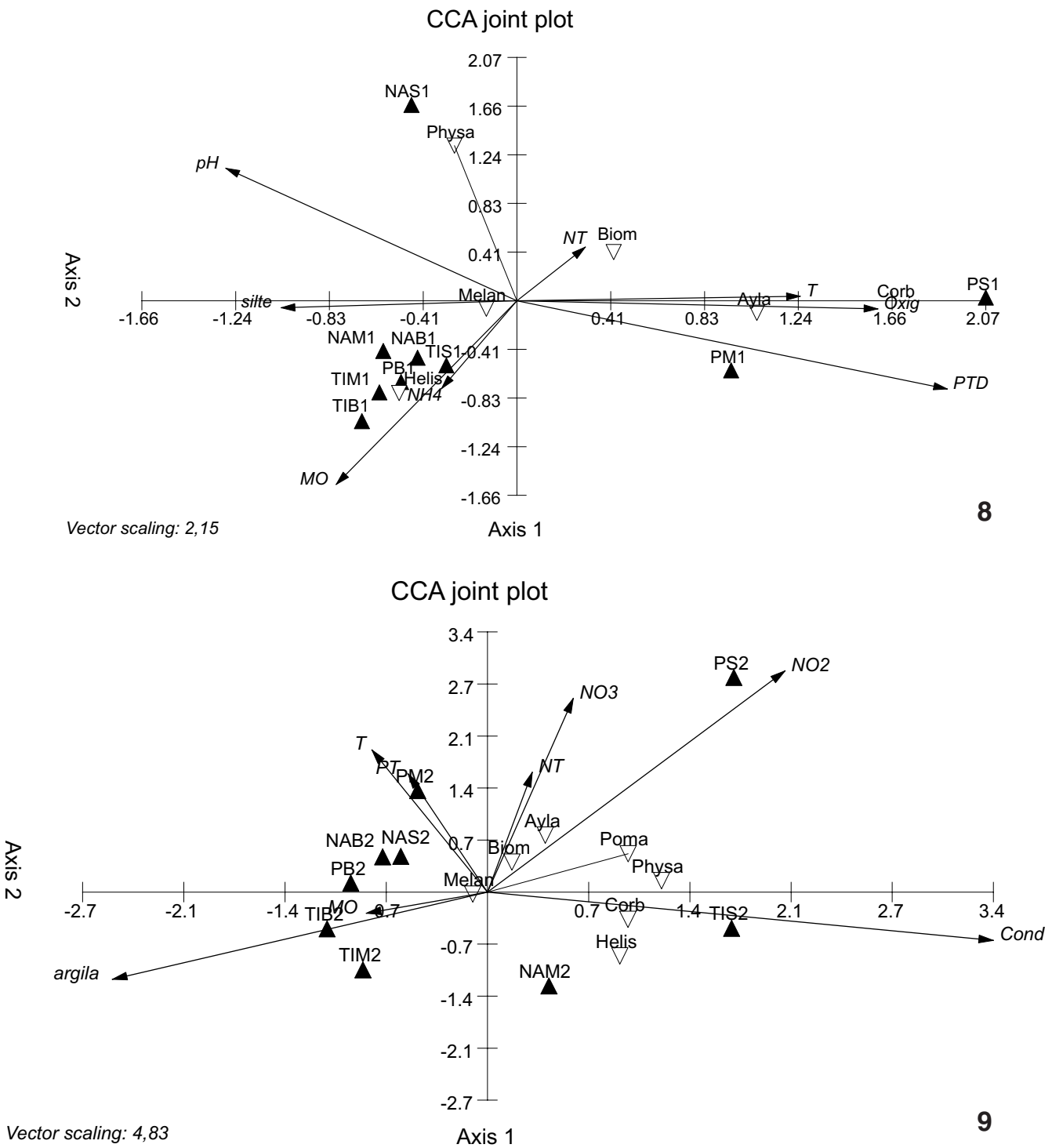

Figuras 8-9. Diagrama de ordenação da CCA com todas as espécies de moluscos e as variáveis ambientais registradas em novembro de 2002 (8) e em agosto de 2003 (9). Physa (Physa cubensis), Biom (Biomphalaria spp.), Ayla (Aylacostoma tenuilabris), Helis (Helisoma sp.), Mel (Melanoides tuberculata), Corb (Corbicula fluminea), P (Promissão), NA (Nova Avanhandava), TI (Três Irmãos), B (Barragem), M (Meio), S (Superior) e 1 (período chuvoso). (MO) matéria orgânica, (T) temperatura, (Cond.) condutividade, (PT) fósforo total, (PTD) fósforo total dissolvido, (NT) nitrogênio total, (NO2) nitrito, (NO3) nitrato, (NH4) amônio, (Oxig) oxigênio dissolvido.

ciada a nenhuma variável ambiental em particular, ocorrendo em todos os gradientes ambientais.

\section{DISCUSSÃO}

As populações dos moluscos nativos nas represas do baixo rio Tietê foram provavelmente afetadas pela recente invasão por espécies exóticas, especialmente pela espécie M. tuberculata. Contudo, devido à ausência de estudos anteriores para a comunidade bentônica destes reservatórios, não é possível avaliar se ocor- reram alterações seja na composição ou na densidade das populações. Os resultados obtidos no presente estudo fornecem apenas uma avaliação preliminar sobre a ocorrência e distribuição dos moluscos nos reservatórios, pelo fato de abordarem apenas um tipo de substrato, o sedimento bentônico, podendo não refletir toda a ocorrência dos moluscos nestes corpos deágua. Como evidenciado por FREITAS (1976) para Biomphalaria glabrata, alguns moluscos podem ocorrer em substratos variados, incluindo a vegetação aquática, além de responderem aos fatores sazonais e 
ao estágio sucessional do ecossistema, levando a amplas variações em densidades populacionais.

Por meio da análise de correspondência canônica (ACC) há indicações de que M. tuberculata é uma espécie bastante plástica, pois ela não se associou com nenhuma variável em particular, não respondendo, portanto, aos gradientes ambientais e distribuindo-se por todos os reservatórios e porções destes. A superioridade desta espécie pode ser explicada pelo fato dela ser altamente competitiva, apresentando características como: predominância de jovens durante todo o ano; baixa taxa de mortalidade; alta capacidade migratória e de dispersão e a capacidade de se estabelecer em todos os tipos de região litorânea e de substratos (FreitAs et al. 1987).

O impacto da espécie exótica M. tuberculata sobre as espé cies nativas tem sido demonstrado em al guns estudos. GIovaneluI et al. (2001) observaram que em Sumidouro (Minas Gerais) esta espécie reduziu substancialmente as populações de B. glabrata; em Betim e em Prudente de M oraes, GuIMARãEs et al. (2001) registraram marcada redução em populações de B. glabrata e B. straminea (Dunker, 1848) em dois lagos depois de sua introdução, seguido pelo completo desaparecimento dessas espécies oito anos depois. Fernandez et al. (2001) também documentaram o deslocamento e declínio das populações de B. glabrata e Pomacea lineata (Spix, 1827) em um pequeno córrego no campus da Fundação Oswaldo Cruz (Rio de Janeiro). Segundo Fernandez et al. (2003), dados preliminares indicaram que as populações nativas de A. tenuilabris, previamente abundantes no Rio Tocantins, tem sido deslocadas por populações densas de M. tuberculata.

O fato dos moluscos nativos A. tenuilabris, B. glabrata, B. intermedia, P. cubensis eP. canaliculata estarem relacionados com diferentes variáveis ambientais em cada um dos períodos amostrados, demonstrou que a partir dos dados obtidos no presente estudo não foi possível estabelecer que característica ou variável ambiental esteve diretamente relacionada com essas espécies ou que fator, ou combinação de fatores, realmente determinou a presença e abundância das mesmas, sugerindo que estudos biológicos mais aprofundados são ainda necessários.

As demais espécies exóticas foram mais abundantes em alguns locais do que as espécies nativas, porém bem menos abundantes do que $M$. tuberculata. Com relação a C. fluminea, assim como $M$. tuberculata, esta também é considerada uma espécie invasora devido à sua grande capacidade de adaptação e grande proliferação em diferentes tipos de ambientes (STRAYER 1999). Segundo MACMAHON (1983), em ambientes lênticos, C. fluminea ocorre principalmente em águas bem oxigenadas, fato também evidenciado neste estudo pela análise de correspondência canônica no período chuvoso. Deve-se ressal tar que esta espécie ocorre preferencial mente em ambientes lóticos (MORTON 1982) e que a construção dos reservatórios por si só ocasiona grandes mudanças na estrutura e função do ecossistema, incluindo grandes al terações nas comunidades (Tundısl et al. 1999).

$O$ bivalve $C$. fluminea é também uma espécie altamente invasora. MAnsur et al. (2004) reportam diversos estudos (MANSUR
\& Garces 1988, Darrigran 1991, Focht \& Veitenheimer-Mendes 2001) onde cerca de cinco anos após a invasão a densidade desta espécie superou a das espécies nativas. Ainda no Brasil, em TAKEDA et al. (2004) observaram um decréscimo acentuado das espécies nativas na planície aluvial do Alto Rio Paraná, após a invasão por C. fluminea, demonstrando que esta espécie exerce efeito adverso sobre as espécies nativas, ocasionando diminuição destas populações por mecanismos ainda não reconhecidos.

Com relação a Helisoma, trata-se de um gênero que não é nativo do Brasil e muito pouco é conhecido sobre sua presença no país. PARAense (1976) registrou a ocorrência de uma população natural de H. duryi (Wetherby 1879) na região Central do país, onde essa espécie foi introduzida provavelmente por aquaristas através de plantas aquáticas.

Nos reservatórios estudados, pode-se observar que os moluscos exóticos ocorreram em abundância mais el evada do que os nativos, evidenciando o alto poder de invasão de $M$. tuberculata, dominante em todos os reservatórios amostrados. O monitoramento nesses ambientes será imprescindível para determinar se as espécies nativas serão eliminadas pelas exóticas ou se persistirão neste sistema, ainda que em baixas densidades populacionais.

\section{AGRADECIMENTOS}

À CAPES, ao Ministério do Meio Ambiente (MMA/BIRD/ GEF) por meio do programa PROBIO, ao CNPq pelo suporte financeiro e aos professores Dr. Wagner E. P. Avelar e Dr. Luiz Ricardo Lopez de Simone, à doutoranda Fernanda Teixeira Marciano e a José Valdecir de Lucca, pelo auxílio em algumas etapas da pesquisa.

\section{REFERÊNCIAS BIBLIOGRÁFICAS}

BASKIN, Y. 1994. Ecosystem function of biodiversity. BioScience 44 (10):657-660.

Cataldo, D. \& D. Boltovskoy. 1999. Population dynamics of corbicula flumine (Bivalvia) in the Paraná River Delta (Argentina). Hydrobiologia 380: 153-163.

CESP.1998. Conservação e manejo nos reservatórios: Limnologia, Ictiologia e Pesca. São Paulo, Companhia Energética de São Paulo, Série Divulgação e Informação, 166p.

CetesB. 2001. Relatório da Qualidade das Águas interiores do Estado de São Paulo. São Paulo, Secretaria do Meio Ambiente, Série Relatórios, 214p.

Darrigran, G.A. 1991. Competencia entre dos especies de pelecípodos invasores, Corbicula fluminea (Mu"ller, 1774) y C. Largillierti (Philippi, 1844), en el litoral Argentino del estuario del Río de La Plata. Biología Acuática 15 (2): 214-215.

Darrigran, G.A.1997. Invasores en la Cuenca del Plata. Ciencia Hoy 7 (38):1-6.

Dudgeon, D. 1986. The life cycle, population dynamics and productivity of Melanoides tuberculata (Müller,1774) (Gastropoda: Prosobranchia: Thiaridae) in Hong Kong. Journal of Zoology 
208: 37-53.

Fernandez, M.A.; S.C. Thiengo \& M.F. Boaventura. 2001. Gastrópodes Límnicos do Campus de Manguinhos. Revista da Sociedade Brasileira de Medicina Tropical 3 (34): 279-282.

Fernandez, M.A.; S.C. Thiengo \& L.R. Simone. 2003. Distribution of the introduced freshwater snail Melanoides tuberculatus (Gastropoda: Thiaridae) in Brazil. The Nautilus 117 (3): 7882.

Focht, T. \& I.L.Veitenheimer-Mendes. 2001. Distribuição de Neocorbicula limosa (Maton) (Bivalvia, Corbiculidae) no lago Guaíba, Rio Grande do Sul, Brasil. Revista Brasileira de Zoologia 18 (1): 35-43.

Freitas, J.R.; L.C. Bedê; P. de Marco JR; L.A. Rocha \& M.B.L. SanTos. 1987. Population dynamics of aquatic snails in Pampulha Reservoir. Memórias do Instuto Oswaldo Cruz 82 (Supl): 299-305.

Giovanelli, A.; C.L.P.A. Silva; L. Medeiros \& M.C. Vasconcellos. 2001. The molluscicidal activity of the latex of Euphorbia splendens var. hislopii on Melanoides tuberculata (Thiaridae), a snail associated with habitats of Biomphalaria glabrata (Planorbidae). Memórias do Instuto Oswaldo Cruz 96 (1): 123-125.

Golterman, H.L.; R.S. Clymo \& M.A.M. Ohstad. 1978. Methods for physical and chemical analysis of freshFwaters. Oxford, Blackwell Scientific Publications, $2^{\text {nd }}$ ed., 213p.

Guimarães, C.T.; C.P. Souza \& D.M. Soares. 2001. Possible competitive displacement of planorbids by Melanoides tuberculata in Minas Gerais, Brazil. Memórias do Instituto Oswaldo Cruz 96 (Supl.): 173-176.

ItUARTE, F.C. 1981. Primeira noticia acerca de la introducción de pelecipodos asiaticos en la area rioplatense (Mollusca, Corbiculidae). Neotropica 27 (7): 79-82.

Johnson, L.E. \& J.T. Cartton.1996. Post-establishment spread in large-scale invasions: dispersal mechanisms of the zebra mussel Dreissena polimorpha. Ecology 77 (6): 1686-1690.

Mackereth, F.J.H.; J. Heron \& J.F. TAILING. 1978. Water analysis: some revised methods for limnologists. Kendal, Titus Wilson \& Son, $+117 p$.

Mansur, M.C.D. \& L.M.M.P. GARCES. 1988. Ocorrência e densidade de Corbicula fluminea (Müller, 1774) e Neocorbicula limosa (Maton, 1811) na Estação Ecológica do Taim e áreas adjacentes, Rio Grande do Sul, Brasil. Iheringia, Série Zoologia (69): 99-116.

Mansur, M.C.D.; C.T. Callil; F.R. Cardoso \& J.A.A. Ibarra. 2004. Uma retrospectiva e mapeamento da invasão de espécies de Corbicula (Mollusca, Bivalvia, Veneroida, Corbiculidae) oriundas do sudeste asiático, na América do Sul, p. 39-58. In: J.S.V. Silva \& R.C.C.L. SouzA. (Eds). Água de lastro e bioinvasão. Rio de Janeiro, Interciência, 224p.

MCMAHON, R.F. 1983. Ecology of an invasive pest bivalve: Corbicula, p. 505-561. In: W.D. RusselL-Hunter (Ed.). The Mollusca. New York, Academic Press, vol. 6, 695p.

MoRTON, B. 1982. Some aspects of the population structure and sexual strategy of Corbicula of fluminalis (Bivalvia: Corbiculidae) from the Pearl River, People's Republic of China. The Journal of Molluscan Studies 48 (1): 1-23.

Paraense, W.L. 1976. A natural population of Helisoma duryi in Brazil. Malacologia 15 (2): 369-76.

Pointier, J.P.; A. Théron \& G. Borel. 1993. Ecology of the introduced snail Melanoides tuberculata (Gastropoda: Thiaridae) in relation to Biomphalaria glabrata I in the marshy forest zone of Guadaloupe, French West Indies. Journal of Molluscan Studies 59: 421-428.

RICCIARDI, A. \& H.J. MACISAAC. 2000. Recent mass invasion of the North American Great Lakes by Ponto-Caspian species. Trends in Ecology and Evolution 15 (2):62-65.

Rocha, O.; K.S. Tavares; M.B.C. Branco; P.A.Z. Pamplin; E.L.G. EsPíndola \& M. MARCheSE. 2006. Padrões de distribuição em reservatórios e relações com o processo de eutrofização, p. 353-372. In: J.G. Tundisl; T. MatSumura-Tundisı \& C.S. GALLI (Ed.). Eutrofização na América do Sul: causas, conseqüências e tecnologias para gerenciamento e controle. São Carlos, Instituto Internacional de Ecologia de São Carlos, $532 p$.

SIMBERLOFF, D. 1996. Impacts of introduced species in the United States. Consequences 2 (2): 13-24.

Simberloff, D. 2001. Eradication of island invasives: practical actions and results achieved. Trends in Ecology and Evolution 16 (6): 273-274.

Strayer, D.L. 1999. Effects of alien species on freshwater mollusks in North America. Journal of the North American Benthological Society 18 (1): 74-88.

Suguio, K. 1973. Introdução à sedimentologia. São Paulo, Blücher, 317p.

TAKEDA, A.M.; D.S. FujITA \& H.M. Fontes. 2004. Perspectives on Exotic bivalves Proliferation in the Upper Paraná River Floodplain, p. 97-100. In: A.A. Agostinho; L. Rodrigues; L.C. Gomes; T.S. MAGela \& L.E. MIRANDA, (Eds). Structure and functioning of the Paraná River and its floodplain. Maringá, EDUEM, 275p.

Thом freshwater snails of Florida. Gainesville, Museum of $\mathrm{Na}$ tural History, University of Florida, 94p. Available in the World Wide Web at: http://www.flmnh.ufl.edu/natsci/ malacology/fl-snail/snails1.htm [Accessed in 17/VIII/2004].

TundisI, J.G.; T. Matsumura-Tundisl; M.C. Calijuri \& E.M.L. Novo. 1991. Comparativelimnology of five reservoirs in the middle Tietê River, São Paulo State. Verhandlungen International Verein Limnology 24 (5) 1489-1496.

Tundisl, J.G.; T. Matsumura-Tundisl \& O. Rocha. 1999. Ecossistemas de Águas Interiores, In: A.C. Rebouças; B. Braga \& J.G. TUnDISI. (Eds). Águas doces no Brasil. Capital ecológico, uso e conservação. São Paulo, Escrituras, 2a ed., 704p.

Vaz, J.F.; H.M.S. Teles; M.A. Correa \& S.P.S. Leite. 1986. Ocorrência no Brasil de Thiara (Melanoides) tuberculata (O.F. Müller, 1774) (Gastropoda, Prosobranchia), primeiro hos-

Revista Brasileira de Zoologia 24 (1): 41-51, março 2007 
pedeiro intermediário de Clonorchis sinensis (Cobbold, 1875) (Trematoda, Plathyhelmintes). Revista de Saúde Pública 20 (4) 318-33.

Veitenheimer-Mendes, I. 1981. Corbicula manilensis (Philippi, 1844) molusco asiático, na bacia do rio Jacuí e do Guaíba, Rio Grande do Sul, Brasil (Bivalvia: Corbiculidae). Iheringia,
Série Zoologia 60: 63-74.

WELCH, P.S. 1948. Limnological methods. Philadelphia, Blakistonco, 381p.

W еLсомме, R.L. 1988. International introductions of inland aquatic species. Rome, FAO Fisheries Technical Paper no. 294, $318 p$.

Recebido em 08.III.2006; aceito em 26.II.2007. 